\title{
Influence of sucrose concentration in the culture medium on the condition of the photosynthetic apparatus of grapes cultured in vitro
}

\author{
Maria Sundyreva ${ }^{1 *}$, Anton Rebrov ${ }^{2}$, and Alisa Mishko ${ }^{1}$ \\ ${ }^{1}$ Federal State Budget Scientific Institution «North Caucasian Regional Research Institute of \\ Horticulture, Viticulture, Wine-making», 40 - Years of Victory, 39, Krasnodar, 350901, Russia \\ ${ }^{2}$ Federal State Budget Scientific Institution All-Russian Research Institute named after Ya.I. \\ Potapenko for Viticulture and Winemaking - Branch of Federal State Budget scientific Institution \\ «Federal Rostov Agricultural Research Center», Novocherkassk, Russia
}

\begin{abstract}
An influence of different sucrose concentrations in the culture media on the photosynthetic parameters, photosynthetic apparatus related genes expression, oxidative processes and acclimation of grape plants cultured in vitro was examined in this article. An increase of the sucrose concentration in the culture media resulted in a reduced expression of several photosynthetic genes. The most effective functioning of the photosynthetic apparatus was discovered by a decreased amount of surcose in culture media. An increase of the sucrose concentration in the culture media disrupts pigments synthesis, particularly carotenoids, which can be a cause of the secondary oxidative stress formation and grape plants growth reduction during acclimation.
\end{abstract}

\section{Introduction}

Clonal micropropagation is a promising method for obtaining healthy planting material, replicating the required number of plants in a short time. In vitro plants are grown in certain conditions: high relative humidity, low light, constant temperature, increased sugar concentrations in the culture media, growth regulators and nutrients, which countribute to the plant growth [1]. Plantlets lose their transpiration regulating mechanisms while adapting to the in vitro environment single [2], resulting in an abnormal anatomy and leaf morphology, poor granas development [3] and low photosynthesis rates [4]. Such deviations lead to the rapid desiccation of micropropagated plantlets when exposed to reduced relative humidity [5]. Sucrose usually serves as a carbon source during clonal micropropagation and ensures in vitro plants growth and development. However, it also inhibits photosynthesis and makes it more difficult to transfer plants from the in vitro to the ex vitro. Survival of plants grown in the highly enriched media depends on the accumulated carbohydrates catabolism during the first two weeks after transfering into the ex vitro conditions. Photoinhibitions occurs during plantlets transferring in the greenhouse conditions. It induces an oxidative stress, which can increase plant death rate $[7,8]$.

\footnotetext{
${ }^{*}$ Corresponding author: mari.sundy@bk.ru
} 


\section{Materials and Methods}

Microcuttings of $1 \mathrm{~cm}$ long with one node of Kandavasta grape variety were used in experiment. Cuttings were cultured in the basal MS medium. The medium was supplemented with 5, 10, 20 and $30 \mathrm{~g} / 1$ sucrose. Each variant included 50 micropropagated plantlets. Plantlets were grown at $23-24{ }^{\circ} \mathrm{C}$ and 2000-2500 lx. Cultivation lasted for 8 weeks, during which the physiological and biochemical parameters of plantlets were estimated. 30 days after planting the adaptation process of the plants to ex vitro conditions was estimated. Provided data comprised of mean values of three experiments.

Analyses were carried out at 28 days of cultivation (28DC), 56 days of cultivation (56DC) and 14 days of acclimatization (14DA). Quantum yield of photosynthesis was measured using PAM fluorometry in light adaptive leaves [9]. Hydrogen peroxide concentration was evaluated according to the FOX1 method [10]. Malonic dialdehyde (MDA) content measured spectrophotometrically by the reaction with thiobarbituric acid according to Heath R.L. и Packer L. (1968). Pigments concentration was determined in the acetone extract according to H.K. Lichtenthaler and C. Buschmann (2001). Analysis of gene expression was conducted using RT-PCR reaction on the CFX96 Touch Real-Time PCR Detection System (BioRad, USA) with commercial kit qPCRmix-HS SYBR according to the manufacturer recommendations (Evrogen, Russia). Glyceraldehyde 3-phosphate dehydrogenase served as a reference gene. Relative gene expression level was calculated using $\Delta \mathrm{Ct}$ method ( $\mathrm{T}$. Schmittgen, K. Livak, 2008). Sequences of the primers used in the analysis are as follows: Light harvesting complex (Lhcb6) F - AACTTCTGCTGCTGTGTTGAATG, R CGACGACAATGAGCCTCCTG [11]; Rubisco-activase (RuAc) F ACAAGGCAGTGGTGGTGTTAGC, R - GACGGAGGTGATGTCGGTGTTG [12]; Chloroplast ATP synthase (ATPsy) F - CGCCAAGAATGTGAGAATCAAGAC, R GAACCGCCACTTCCATACCTG [11]; Glyceraldehyde 3-phosphate dehydrogenase (GAPDH) F CCACAGACTTCATCGGTGACA, R - TTCTCGTTGAGGGCTATTCCA [13].

\section{Results and Discussion}

An increased content of the photosynthetic pigments alongside with the elevated sucrose concentration in the culture media was established in 28 DC plantlets, which can be linked to the increased availability of organic compounds. However, chlorophyll content in 56DC plantlets, cultivated with high sucrose concentration in the culture media, was lower than that in the media with $5 \mathrm{~g} / 1$ sucrose. A similar tendency continued during the period of plants acclimation, however, total pigments content was significally higher, which is related to the autotrophic nutrition induction (fig. 1).

56DC plantlets were characterized by the high $\mathrm{Chl} \mathrm{A/B}$ ratio. After transfering into the ex vitro conditions chlorophyll $\mathrm{B}$ content increased and the ratio decreased respectively.

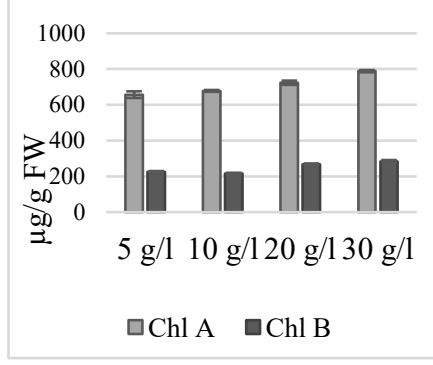

$28 \mathrm{DC}$

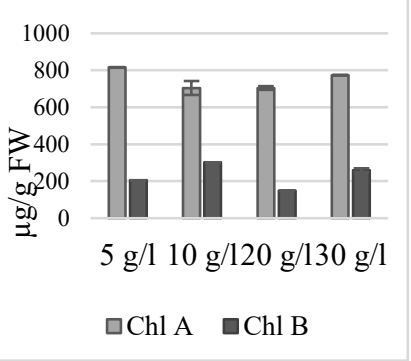

56 DC

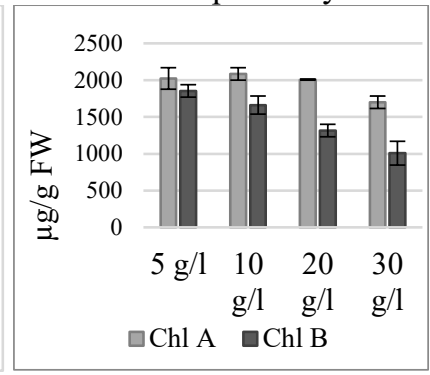

14 DA

Fig. 1. Chlorophyll content in grape leaves 
Carothenoids are the most important detoxification elements of the reactive oxygen species (ROS) which are generated in the chloroplasts. Carothenoids content in the grape plantlets raised with the increase of the sucrose concentration in the culture media on the first month of the in vitro cultivation. In later periods 20 and $30 \mathrm{~g} / 1$ of sucrose in the culture media resulted in a decline of the carothenoids concentration (fig. 2).
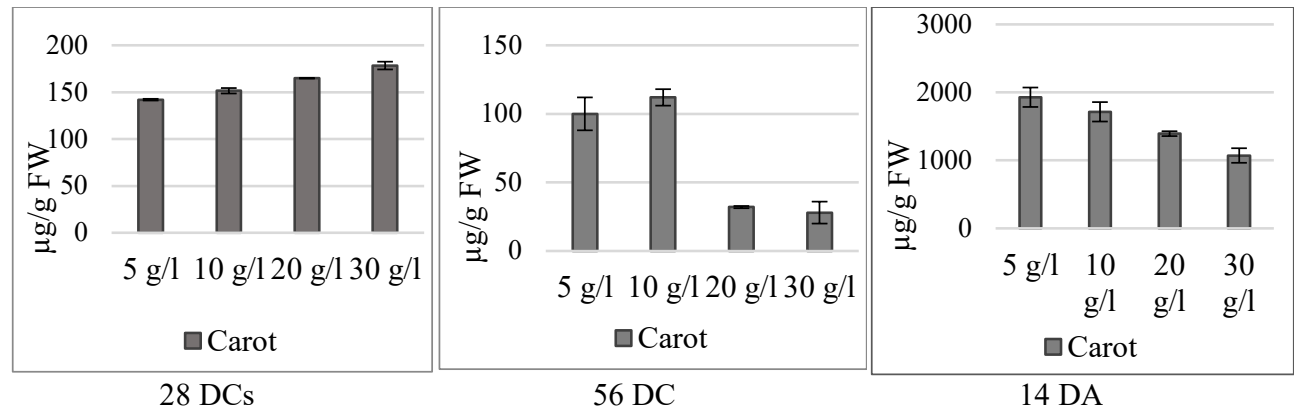

Fig. 2. Carotenoid content in grape leaves

In 56DC grape plantlets increased sucrose concentration in the culture media inhibited expression of ATPsy, LHCb6, RuAc. It was shown, that expression of the nuclear photosynthetic genes is substantially inhibited with the increase of the soluble carbohydrates in the cytoplasm [14-16]. The most significant decrease of genes expression has been already observed at $10 \mathrm{~g} / \mathrm{l}$ of sucrose in the culture media. Expression of LHCb6 decreased gradually as the concentration of the sucrose in the culture media increased. Expression of RuAc and chloroplast ATPsy, which are directly involved in the $\mathrm{CO}_{2}$ assimilation, was minimum at 10 $\mathrm{g} / \mathrm{l}$ of sucrose, and slightly increased at 20 and $30 \mathrm{~g} / \mathrm{l}$. The level of these genes expression is well correlated, which is explained by dependence of the RuAc expression activity on the ATP content [17]. A low-enriched culture medium was necessary at the stage of in vitro rooting for subsequent good development and the photosynthetic apparatus of grape plantlets (fig. 3).
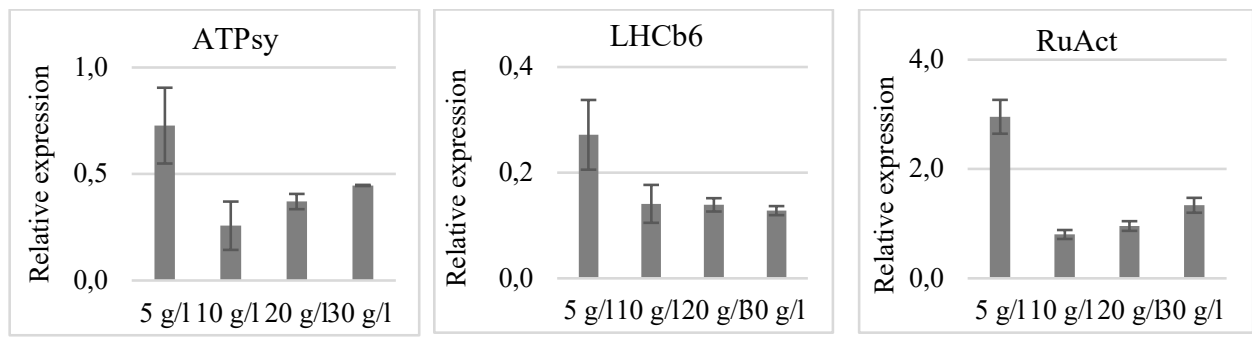

Fig. 3. Photosynthesis related genes expression, $56 \mathrm{DC}$

Quantum yield of photosynthesis (QY) in light adaptive leaves declined with the increase of the sucrose concentration in the culture media. Plantlets cultured on the media with 5 and $10 \mathrm{~g} / \mathrm{l}$ of sucrose were characterized by higher QY. 56DC and 14DA plantlets cultured on the media with 20 and $30 \mathrm{~g} / \mathrm{l}$ of sucrose were characterized by a decreased QY. Decline of the QY can be associated with the induction of the stress condition as well as with the inhibition of the photosynthetic processes by sucrose (data not shown). 
Plantlets cultivated on the low-enriched media easily change over to autonomous autotrophic nutrition, grow and develop [6]. It was shown that the low sucrose content in the culture media promotes a substantial increase of pigments content and enchances functional characteristics of antennas and light harvesting complexes [18], which is consistent with our results.

A poorly formed photosynthetic apparatus cannot provide normal donor-acceptor relationships between plant organs, which enhances the formation of ROS and reduces plant resistance to environmental conditions.

Oxidative stress, estimated on the basis of MDA content, was formed more intensively in plantlets grown at high concentrations of sucrose in culture media. This result is consistent with a reduced leaf carotenoid content and a reduced quantum yield of the photosynthesis. Generally, MDA content was significantly higher in plantlets in ex vitro conditions than in in vitro cultured plantlets (fig. 4).

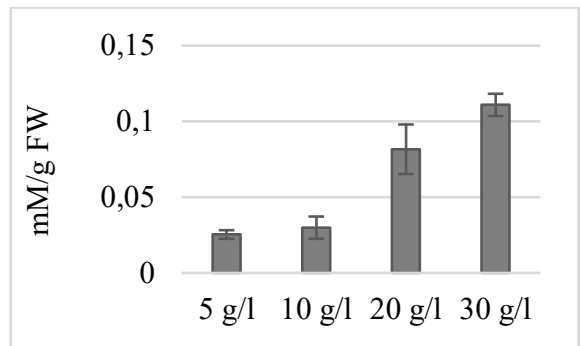

$56 \mathrm{DC}$

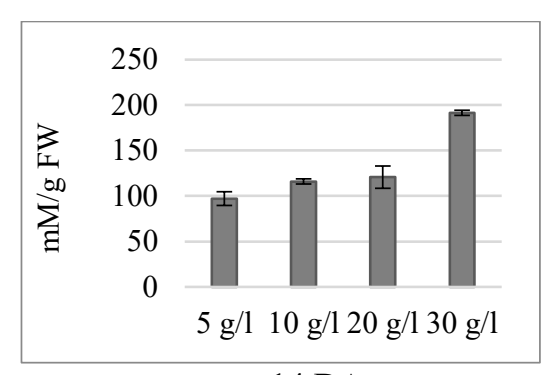

14 DA

Fig. 4. MDA content in grape plantlets

Plants cultivated on the highly-enriched media had the highest biomass before transferring into ex vitro conditions. The contribution of the plant's vegetative organs in the total mass varied. Thus, an increase of the sucrose concentration in the culture media resulted in a more intense growth of roots. Difference of shoots length between experimental variants was insignificant (fig. 5).

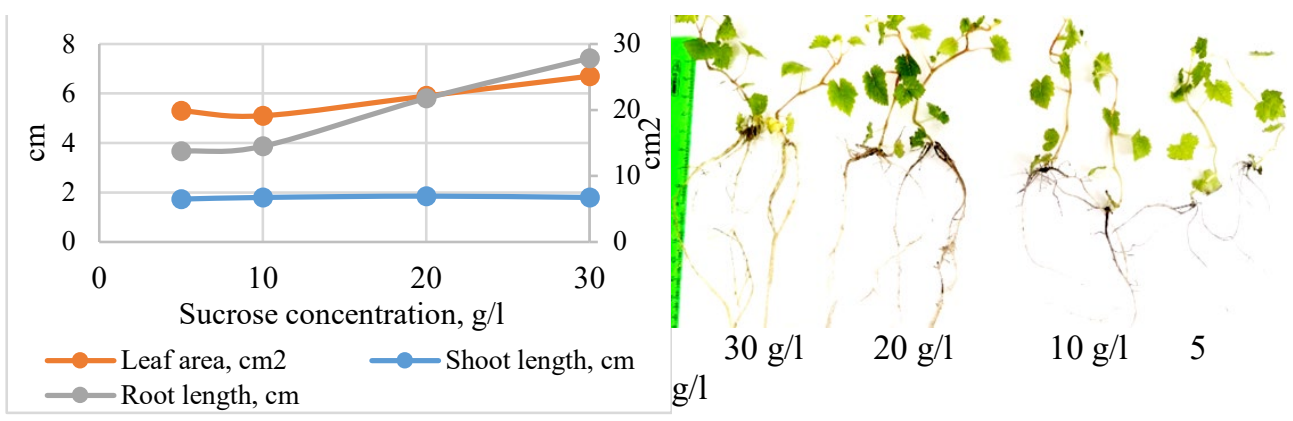

Fig .5. Biometric characteristics of grape plantlets cultured in vitro

There were no significant differences between plantlets establishment during transferring into ex vitro conditions. However, a positive effect of low sucrose concentration in culture media on ex vitro plantlets development should be noted (table 1).

Table 1. The influence of various sucrose concentrations in the culture medium on the acclimatization of grape plantlets to ex vitro conditions (30 days of acclimatization)

\begin{tabular}{l|l} 
Development indicators & Variants \\
\hline
\end{tabular}




\begin{tabular}{|c|c|c|c|c|}
\hline & $5 \mathrm{~g} / 1$ & $10 \mathrm{~g} / 1$ & $20 \mathrm{~g} / 1$ & $30 \mathrm{~g} / 1$ \\
\hline Plant height, $\mathrm{cm}$ & $15,0 \pm 1,7$ & $11,3 \pm 1,7$ & $12,1 \pm 1,8$ & $10,4 \pm 1,6$ \\
\hline Number of leaves, pcs. & $9,3 \pm 1,3$ & $6,2 \pm 0,6$ & $6,0 \pm 0,8$ & $5,1 \pm 0,8$ \\
\hline Number of internodes, pcs. & $11,5 \pm 0,8$ & $8,9 \pm 1,1$ & $7,7 \pm 1,1$ & $6,6 \pm 1,0$ \\
\hline Internode length, $\mathrm{cm}$ & $1,3 \pm 0,13$ & $1,3 \pm 0,17$ & $1,6 \pm 0,07$ & $1,4 \pm 0,33$ \\
\hline Leaf surface, $\mathrm{cm} 2$ & $79,5 \pm 17,1$ & $40,1 \pm 8,4$ & $47,2 \pm 13,4$ & $39,6 \pm 5,9$ \\
\hline $\begin{array}{l}\text { Number of roots observed on the } \\
\text { walls of transparent pots, pcs. }\end{array}$ & $14,2 \pm 3,4$ & $17,1 \pm 3,5$ & $15,6 \pm 4,6$ & $15,4 \pm 5,1$ \\
\hline Adaptability, score & $4,3 \pm 0,17$ & $4,0 \pm 0,21$ & $4,1 \pm 0,23$ & $3,8 \pm 0,7$ \\
\hline Survival, $\%$ & $\begin{array}{r}100,0+0,0 \\
-13,3\end{array}$ & $\begin{array}{r}100,0+0,0 \\
-13,3 \\
\end{array}$ & $\begin{array}{r}100,0+0,0 \\
-13,3\end{array}$ & $\begin{array}{r}96,0+3,3 \\
-15,5\end{array}$ \\
\hline
\end{tabular}

After 1 month of cultivation in greenhouse conditions plantlets cultured with $5 \mathrm{~g} / \mathrm{l}$ sucrose, were superior to other experimental variants in terms of growth characteristics. Hence, a deficiency of growth processes in the in vitro plantlets, cultivated on the lowenriched media, was mitigated by a reduced oxidative stress and more developed photosynthetic apparatus

\section{Conclusions}

Low-enriched media facilitated more effective formation of the photosynthetic apparatus of grape's plantlets at rooting stage. Content of photosynthetic pigments and quantum yield PSII declined with the increase of the sucrose concentraiton in the culture media. Weakening of the photosynthetic apparatus formation associated with the reduced expression of the Rubisco-activase, light harvesting complex proteins, chloroplast ATP-synthase genes, which was caused by an increased sucrose concentration. Plants, grown on the high-enriched media, were characterised by the presence of a more intense oxidative stress, which associated with both with the osmotic stress and with the disruption of the photosynthetic apparatus formation.

Plants with the highest biomass, obtained on the culture media with 20 and $30 \mathrm{~g} / \mathrm{l}$ sucrose, didn't bring any benifits to the growth in the greenhouse conditions, which is linked to the unbalanced physiological state. For the development and formation of the effective photosynthetic apparatus and further ex vitro growth and development the application of the low-enriched media on the plant rooting stage is recommended.

Acknowledgments. The reported study was funded by RFBR and Krasnodar Region according to the research project № 19-44-230037 r-a.

\section{References}

1. J. Mao, W. Li, B. Mi, M. M. Dawuda, A. Calderón-Urrea, Z. Ma, Y. Zhang, B. Chen, Planta, 246, 537-552 (2017). https://doi.org/10.1007/s00425-017-2712-X

2. Z. Maleki, K. Vahdati, S. Aliniaeifard, Sc. Hort., 224, 1-7 (2017). https://doi.org/10.1016/J.SCIENTA.2017.04.023

3. S. Dutta Gupta, B. Jatothu, Plant Biotechnol, 7, 211-220 (2013). https://doi.org/10.1007/s11816-013-0277-0

4. P. L. Sáez, L. A. Bravo, K. L. Sáez, M. Sánchez-Olate, M. I. Latsague, D. G. Ríos1, Biol Plant, 56, 15-24 (2012). https://doi.org/10.1007/s10535-012-0010-9

5. S.Y. Park, H.K. Moon, H.N. Murthy, Y.W. Kim, Biol. Plant., 55 (3), 559-562, (2011). https://link.springer.com/content/pdf/10.1007/s10535-011-0125-4.pdf 
6. R. Lembrechts, N. Ceusters, M. P. De Proft, J. Ceusters, Sci. Hort., 224, 226-231 (2017). https://doi.org/10.1016/j.scienta.2017.06.015

7. C. Goh, S. Ko, S. Koh, Y.J. Kim, H.J. Bae, J. Plant Biol, 55, 93-101 (2012). https://doi.org/10.1007/s12374-011-9195-2

8. V. Demidchik, Env. and Exp. Bot., 109, 212-228 (2015). https://doi.org/10.1016/j.envexpbot.2014.06.021

9. V. N. Goltsev, M. H. Kalaji, M. A. Kouzmanova, S. I. Allakhverdiev, Variable and Delayed Chlorophyll a Fluorescence - Basics and Application in Plant Sciences, (Moscow-Izhevsk: Institute of Computer Science, (2014). https://www.researchgate.net/publication/299847794_Variable and Delayed_Chloroph yll a Fluorescence-Basics and Application in Plant Sciences

10. J. D. Castaño, J. Zhang, J.S. Schilling, J. Microbiol. Methods, 145, 10-13 (2018). https://doi.org/10.1016/j.mimet.2017.12.004

11. X. Zhao, W. Li, Y. Wang, Z.H. Ma, S.J. Yang, Q. Zhou, J. Mao, B.H. Chen, BMC Plant Biol, 19, 42 (2019). https://doi.org/10.1186/s12870-019-1644-y

12. P. Margaria, S. Palmano, Proteomics, $11 \quad$ (2), 212-224 (2011). https://doi.org/10.1002/pmic.201000409

13. W. Dumin, M. Rostas, C. Winefield, Mol Biol Rep, 45, 263-277 (2018). https://doi.org/10.1007/s11033-018-4159-y

14. A. K. M. Lobo, M. de Oliveira Martins, M. C. L. Neto, E.C. Machado, R.V. Ribeiro, J.A.G. Silveira, J. Plant Physiol., 179, 113-121 (2015). https://doi.org/10.1016/j.jplph.2015.03.007

15. A. Eckstein, P. Zięba, H. Gabryś, J Plant Growth Regul, 31, 90-101 (2012). https://doi.org/10.1007/s00344-011-9222-Z

16. P. Horacio, G. Martinez-Noel, Plant Signal Behav, 8 (3), e23316 (2013). https://doi.org/10.4161/psb.23316

17. J. C. Scales, M. A.J. Parry, M. E. Salvucci, Photosynth Res, 119, 355-365 (2014). https://doi.org/10.1007/s11120-013-9964-5

18. L. P. Pérez, Y. P. Montesinos, J. G. Olmedo, R. R. Sanchez, O. N. Montenegro, R. B. Rodriquez, O. H. Ribalta, R. C. R. Escriba, D. Daniels, R. Gómez-Kosky, Afr. J. Biotech., 14(35), 2622-2635 (2015). https://doi.org/10.5897/AJB2015.14814 
Maria Sundyreva

8 (996) 405-52-73; Mari.sundy@bk.ru

Federal State Budget Scientific Institution «North Caucasian Regional

Research Institute of Horticulture, Viticulture, Wine-making»,

Anton Rebrov

8 (904) 347-64-81; rebrow-anton@yandex.ru

Federal State Budget Scientific Institution All-Russian Research Institute of Viticulture and Winemaking named after Ya.I.. Potapenko, head of laboratory of plant biotechnology

Alisa Mishko

8 (989) 824-15-23; mishko-alisa@mail.ru

Federal State Budget Scientific Institution «North Caucasian Regional Research Institute of Horticulture, Viticulture, Wine-making», researcher of laboratory of plant physiology and biochemistry 